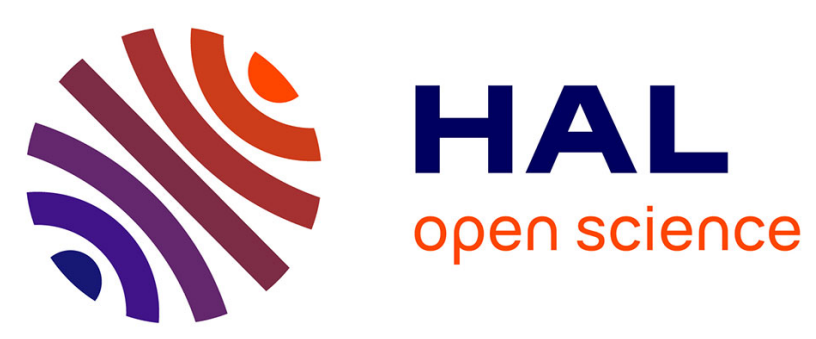

\title{
From cellular to tissue scales by asymptotic limits of thermostatted kinetic models
}

Carlo Bianca, Christian Dogbe, Annie Lemarchand

\section{To cite this version:}

Carlo Bianca, Christian Dogbe, Annie Lemarchand. From cellular to tissue scales by asymptotic limits of thermostatted kinetic models. The European Physical Journal Plus, 2016, 131 (2), pp.41. 10.1140/epjp/i2016-16041-7 . hal-01323803

\section{HAL Id: hal-01323803 https://hal.sorbonne-universite.fr/hal-01323803}

Submitted on 31 May 2016

HAL is a multi-disciplinary open access archive for the deposit and dissemination of scientific research documents, whether they are published or not. The documents may come from teaching and research institutions in France or abroad, or from public or private research centers.
L'archive ouverte pluridisciplinaire HAL, est destinée au dépôt et à la diffusion de documents scientifiques de niveau recherche, publiés ou non, émanant des établissements d'enseignement et de recherche français ou étrangers, des laboratoires publics ou privés. 


\title{
From cellular to tissue scales by asymptotic limits of thermostatted kinetic models
}

\author{
Carlo Bianca $^{1,2}$, Christian Dogbe ${ }^{5}$ and Annie Lemarchand ${ }^{3,4}$ a \\ 1 Ecole Normale Supérieure, Laboratoire de Physique Statistique, 24, rue Lhomond, 75231 Paris cedex 05, France \\ 2 CNRS, UMR 8550, LPS, Paris, France \\ 3 Sorbonne Universités, UPMC Univ Paris 06, UMR 7600, Laboratoire de Physique Théorique de la Matière Condensée, 4, place Jussieu, case \\ courrier 121, 75252 Paris cedex 05 , France \\ 4 CNRS, UMR 7600 LPTMC, Paris, France \\ 5 Université de Caen, Department of Mathematics, LMNO, CNRS, UMR 6139, 14032 Caen Cedex, France
}

Received: date / Revised version: date

\begin{abstract}
Tumor growth strictly depends on the interactions occurring at the cellular scale. In order to obtain the linking between the dynamics described at tissue and cellular scales, asymptotic methods have been employed, consisting in deriving tissue equations by suitable limits of mesoscopic models. In this paper, the evolution at the cellular scale is described by thermostatted kinetic theory that include conservative, nonconservative (proliferation, destruction and mutations), stochastic terms, and the role of external agents. The dynamics at the tissue scale (cell-density evolution) is obtained by performing a low-field scaling and considering the related convergence of the rescaled framework when the scaling parameter goes to zero.
\end{abstract}

PACS. 02.30.Jr Partial differential equations - 02.50.Cw Kinetic theory - 02.60.Nm Integral and integrodifferential equations

\section{Introduction}

Tumor disease has recently attracted the attention of many scientists, including physicists, applied mathematicians and bioinformatics researchers. The mechanisms which are responsible of tumor growth comprises many phenomena occurring at different scales, among others, molecular, cellular and tissue scales, see [1]. In particular at molecular scale (length scales of the order of $\mathrm{nm} \sim \mathrm{mm}$ and time scales of $\mathrm{ns} \sim \mathrm{s}$ ) the dynamics of populations of proteins, peptides, and lipids are studied [2,3]. The cellular scale (length scales of the order of $\mathrm{mm}$ and time scales of min $\sim$ hour) is concerned with the description of the malignant transformation of normal cells, cell-cell interactions, the heterogeneous tumor environment [4]. The tissue scale (length scales of the order of $\mathrm{mm} \sim \mathrm{cm}$ and time scales of day $\sim$ year) focuses on the dynamics of the gross tumor behavior including morphology, shape, extent of vascularization, and invasion, under different environmental conditions (cells can be treated as a single continuum) [5]. Therefore a complete understanding of tumor formation requires a multiscale approach. In particular the description of transport phenomena are of great importance in the tumor onset.

At the molecular scale, the dynamics of individual molecules is followed, for example by molecular dynamics simulations. Mean-field approaches to phenomena at cellular scale are based on ordinary differential equations (ODE), see the review paper [6]. More refined descriptions rely on generalized kinetic theory theory [7] and agent-based approach [8] which provide the evolution of cell distribution function. Tumor growth at tissue scale is usually described by employing continuum mechanics approach, which allows to obtain conservation laws for spatiotemporally-varying densities of different cell types, see books [9] and the references cited therein. However in order to have a complete description of tumor disease and for understanding the different phenomena occurring at the different scales that have as results tumor metastasis, it is fundamental to link the dynamics described by the different approaches developed at the different scales (multiscale approach). The multiscale approach requires the development of mathematical methods, possibly a theory, that transfer information from the lower to the upper scale. A first attempt to the multiscale approach has been proposed by applied mathematicians in kinetic theory of gases in particular for linking the information between the mesoscopic (kinetic) scale and the macroscopic scale. The mathematical method, called asymptotic method, consists in deriving macroscopic equations by suitable limits of Boltzmann-type equations for distributions

\footnotetext{
Send offprint requests to:

a Present address: bianca@lps.ens.fr, christian.dogbe@unicaen.fr, anle@1ptmc.jussieu.fr
} 
of molecule positions and velocities, see, among others, [10-17]. Then asymptotic methods have been developed and employed for deriving macroscopic equations in tumor disease, see the reviews [18,19].

The asymptotic method comprises three main steps: the assessment of time-space scalings (low-field or high field scaling), the derivation of the rescaled equation in terms of the scaling parameter, the asymptotic limit (under suitable technical assumptions) when the scaling parameter goes to zero. According to the scaling, the macroscopic equation consists of a parabolic (driftdiffusion) or hyperbolic equation, see papers [20-24] and the references cited therein.

Recently, the thermostatted kinetic theory has been proposed for modeling complex systems in physics and life sciences and has been generalized to systems with mutations in paper [25] for the modeling of tumor-immune-system competition [26,27]. Differently from classical kinetic theory which deals with molecule position and velocity distribution, the thermostatted kinetic theory refers to cells. In this theory, the microscopic state of the cell includes, in addition to classical space and velocity variables, the activity variable which models the ability of a cell to perform specific strategies. Furthermore the thermostatted kinetic theory enables to capture nonequilibrium stationary states that are typical of most complex biological systems, see [28].

This paper deals with the low-field limit of a thermostatted kinetic framework, which includes transitions in the cells strategy, birth/death processes, cell mutations and the role of microscopic external actions, e.g. environment pressure (open systems). The framework also includes the space structure and the stochastic velocity-jump process proposed in [29]. The time evolution of each subsystem is depicted by a distribution function, over the microscopic state of the particles, satisfying an evolution equation. The derived macroscopic equation, which refers to the time evolution of the cell density, shows how the different kind of interactions at the cellular scale may affect tumor tissue growth.

It is worth stressing that the asymptotic method proposed in the present paper is quite general because the method is developed for generic interaction operators that constitute the thermostatted kinetic framework. Moreover, to the best of our knowledge, this is the first time that the low-field scaling is performed for thermostatted kinetic models for open systems. The high-field limit has been recently developed in [30].

The present paper is organized into four more sections, which follow this introduction. In detail, Section 2 focuses on the derivation of the thermostatted kinetic framework for open systems. The framework acts as a background paradigm for the derivation of specific models for tumor-immune-system competition. Section 2 is concerned with the definition of the local quantities and the main properties of the operator modeling the velocity-jump process. Section 3 deals with the derivation of the macroscopic equation for the local density by the low-field scaling. Section 4 is devoted to a specific model of tumor-immunesystem competition under the action of a cellular vaccine. Finally Section 5 concludes the paper with a critical analysis and research perspectives.

\section{The Kinetic Framework at Cellular Scale}

This section is concerned with the mathematical modeling of the tumor growth at cellular scale by thermostatted kinetic theory that acts as a general paradigm for the derivation of specific models. Specifically in the tumor formation a large number of cells (active particles) that interact in nonlinear matter are involved and macroscopic external force fields $\mathcal{F}_{i}=\mathcal{F}_{i}(u): D_{u} \rightarrow \mathbb{R}$ extent an action on the system. The microscopic state of a cell is the triplet $(\mathbf{x}, \mathbf{v}, u)$, which means that the cell at time $t \in[0, \infty)$, is located in $\mathbf{x} \in D_{\mathbf{x}} \subset \mathbb{R}^{d}$ (usually $d=3$ ), with velocity variable $\mathbf{v} \in D_{\mathbf{v}} \subset \mathbb{R}^{d}$ and activity variable $u \in D_{u} \subset \mathbb{R}$. Cells expressing the same function are grouped into a subsystem, called functional subsystem. The evolution of each functional subsystem is depicted by the distribution function $f_{i}(t, \mathbf{x}, \mathbf{v}, u):[0, \infty) \times D_{\mathbf{x}} \times D_{\mathbf{v}} \times D_{u}$, for $i \in\{1,2, \ldots, n\}$, and such that, for any fixed time $t$, the quantity $f_{i}(t, \mathbf{x}, \mathbf{v}, u) d \mathbf{x} d \mathbf{v} d u$ represents the density of cells in the volume element $d \mathbf{x} d \mathbf{v} d u$ centered at $(\mathbf{x}, \mathbf{v}, u)$.

Let $\mathbf{f}=\mathbf{f}(t, \mathbf{x}, \mathbf{v}, u)=\left(f_{1}(t, \mathbf{x}, \mathbf{v}, u), \ldots, f_{n}(t, \mathbf{x}, \mathbf{v}, u)\right)$ be the vector whose components are the distribution functions of the functional subsystems, $\Omega=D_{\mathbf{x}} \times D_{\mathbf{v}} \times D_{u}$ be the domain of all possible microscopic states, $d \Omega=d \mathbf{x} d \mathbf{v} d u$ the Lebesgue measure on $\Omega$ and

$$
\widetilde{f}(t, \mathbf{x}, \mathbf{v}, u)=\sum_{i=1}^{n} f_{i}(t, \mathbf{x}, \mathbf{v}, u) .
$$

The evolution equation for the $i$ th functional subsystem is obtained by equating the time derivative of $f_{i}$ to the balance of the inlet and outlet flows in the elementary volume $d \Omega$. Accordingly we have:

$$
\left(\partial_{t}+\mathbf{v} \cdot \nabla_{\mathbf{x}}\right) f_{i}+\partial_{u}\left(\mathcal{F}_{i}(u)\left(1-u \int_{\Omega} u \tilde{f} d \Omega\right) f_{i}\right)=J_{i}[\mathbf{f}]+v V_{i}\left[f_{i}\right]
$$

where $J_{i}[\mathbf{f}]=J_{i}[\mathbf{f}](t, \mathbf{x}, \mathbf{v}, u)$ is the operator that models the gain-loss of cells due to transitions in the activity variable and it reads:

$$
\begin{aligned}
J_{i}[\mathbf{f}]= & \sum_{j=1}^{n} \eta_{i j} \int_{D_{u} \times D_{u}} \mathcal{A}_{i j}\left(u_{*}, u^{*}, u\right) f_{i}\left(t, \mathbf{x}, \mathbf{v}, u_{*}\right) f_{j}\left(t, \mathbf{x}, \mathbf{v}, u^{*}\right) d u_{*} d u^{*} \\
& -f_{i}(t, \mathbf{x}, \mathbf{v}, u) \sum_{j=1}^{n} \eta_{i j} \int_{D_{u}} f_{j}\left(t, \mathbf{x}, \mathbf{v}, u^{*}\right) d u^{*}
\end{aligned}
$$


where:

- $\eta_{i j}$ models the probability that a cell of the $i$ th functional subsystem with activity $u_{*}$ interacts instantaneously with a cell of the $j$ th functional subsystem with activity $u^{*}$;

- $\mathcal{A}=\mathcal{A}\left(u_{*}, u^{*}, u\right): D_{u} \times D_{u} \times D_{u} \rightarrow \mathbb{R}^{+}$is the density function modeling the probability that cells of the $i$ th functional subsystem with activity $u_{*}$ interacting with cells of the $j$ th functional subsystem with activity $u^{*}$ reach the activity $u$. In particular $\mathcal{A}\left(u_{*}, u^{*}, u\right)$ satisfies the following identity:

$$
\int_{D_{u}} \mathcal{A}\left(u_{*}, u^{*}, u\right) d u=1, \quad \forall u_{*}, u^{*} \in D_{u}
$$

The operator $V_{i}\left[f_{i}\right] \equiv V_{i}\left[f_{i}\right](t, \mathbf{x}, \mathbf{v}, u)$ models the velocity-jump process, and it reads:

$$
V_{i}\left[f_{i}\right]=\int_{D_{\mathbf{v}}}\left[T_{i}\left(\mathbf{v}^{*}, \mathbf{v}\right) f_{i}\left(t, \mathbf{x}, \mathbf{v}^{*}, u\right)-T_{i}\left(\mathbf{v}, \mathbf{v}^{*}\right) f_{i}(t, \mathbf{x}, \mathbf{v}, u)\right] d \mathbf{v}^{*},
$$

where $T_{i}\left(\mathbf{v}^{*}, \mathbf{v}\right)$ is the turning kernel which gives the probability that, if a jump occurs, the velocity $\mathbf{v}^{*} \in D_{\mathbf{v}}$ jumps into the velocity $\mathbf{v} \in D_{\mathbf{v}}$. The domain $D_{\mathbf{v}}$ is assumed to be bounded and spherically symmetric with respect to origin (i.e. $\mathbf{v}$ and $-\mathbf{v} \in D_{\mathbf{v}}$ ). In particular $v$ is the turning rate or turning frequency of the velocity-jump, hence $1 / v$ is the mean run time.

The operator $\mathcal{T}_{\mathcal{F}_{i}}[\mathbf{f}]=\mathcal{T}_{\mathcal{F}_{i}}[\mathbf{f}](t, \mathbf{x}, \mathbf{v}, u)$ is the transport term that models the Gaussian thermostat [31,32], and it reads:

$$
\mathcal{T}_{\mathcal{F}_{i}}[\mathbf{f}]:=\partial_{u}\left(\mathcal{F}_{i}(u)\left(1-u \int_{\Omega} u \widetilde{f}(t, \mathbf{x}, \mathbf{v}, u) d \Omega\right) f_{i}(t, \mathbf{x}, \mathbf{v}, u)\right)
$$

In particular (2.5) is a damping operator adjusted to control

$$
\sum_{i=1}^{n} \int_{D_{u}} u^{2} f_{i}(t, \mathbf{x}, \mathbf{v}, u) d u
$$

\subsection{Proliferative/Desctructive and Mutative Events}

The framework (2.2) can be further generalized by introducing the role of nonconservative processes. Specifically, interactions among the cells may generate proliferation/destruction of other cells (birth-death process). This type of interaction is modeled by the following operator: $N_{i}[\mathbf{f}]=N[\mathbf{f}](t, \mathbf{x}, \mathbf{v}, u)$ :

$$
N_{i}[\mathbf{f}]=f_{i}(t, \mathbf{x}, \mathbf{v}, u) \sum_{j=1}^{n} \alpha_{i j} \int_{D_{u}} f_{j}\left(t, \mathbf{x}, \mathbf{v}, u^{*}\right) d u^{*} .
$$

where $\alpha_{i j}=\eta_{i j} \mu_{i j}$, being $\mu_{i j}$ the net proliferative/destructive rate.

Because of DNA corruptions, cells can become cells of another functional subsystem. These kinds of interactions are modeled by the following operator $M_{i}[\mathbf{f}]=M_{i}[\mathbf{f}](t, \mathbf{x}, \mathbf{v}, u)$ :

$$
M_{i}[\mathbf{f}]=\sum_{h=1}^{n} \sum_{k=1}^{n} \beta_{i h k} \int_{D_{u} \times D_{u}} f_{h}\left(t, \mathbf{x}, \mathbf{v}, u_{*}\right) f_{k}\left(t, \mathbf{x}, \mathbf{v}, u^{*}\right) d u_{*} d u^{*}
$$

where $\beta_{i h k}=\eta_{h k} \varphi_{h k}^{i}$, being $\varphi_{h k}^{i}$ the net mutative rate into the $i$ th functional subsystem, due to interactions that occur with rate $\eta_{h k}$ between the cells $\left(\mathbf{x}, \mathbf{v}, u_{*}\right)$ of the $h$ th functional subsystem and the cells $\left(\mathbf{x}, \mathbf{v}, u^{*}\right)$ of the $k$ th functional subsystem.

Bearing all above in mind, the thermostatted kinetic framework with proliferative/destructive and mutative interactions reads:

$$
\left(\partial_{t}+\mathbf{v} \cdot \nabla_{\mathbf{x}}\right) f_{i}+\mathcal{T}_{F_{i}}[\mathbf{f}]=J_{i}[\mathbf{f}]+N_{i}[\mathbf{f}]+M_{i}[\mathbf{f}]+v V_{i}\left[f_{i}\right]
$$

\subsection{Thermostatted Kinetic Framework for Open Systems}

The previous thermostatted kinetic structures refer to the modeling of tumor growth subjected to external force fields at the macroscopic scale but in the absence of external interactions at the microscopic scale. The modeling of external agents at the microscopic scale is performed by representing the external agents as functional subsystems that have the ability to modify the state $u$ of the system by a particular action related to the variable $\omega \in D_{u}$. Assuming that the ith inner functional subsystem interacts with the $r$ th external agent, for $r \in\{1,2, \ldots, m\}$, and denoting by $g_{\text {ir }}=g_{\text {ir }}(t, \mathbf{x}, \mathbf{v}, \omega):[0, \infty) \times D_{\mathbf{x}} \times D_{\mathbf{v}} \times D_{u} \rightarrow \mathbb{R}^{+}$the 
related distribution function (known function of its arguments), the microscopic external actions are modeled by the following operator $Q_{i}\left[\mathbf{f}, g_{i}\right]=Q_{i}\left[\mathbf{f}, g_{i}\right](t, \mathbf{x}, \mathbf{v}, u)$ :

$$
\begin{aligned}
Q_{i}\left[\mathbf{f}, g_{i}\right]= & \sum_{r=1}^{m} \eta_{i r}^{e} \int_{D_{u} \times D_{u}} \mathcal{B}_{i r}\left(u_{*}, \omega^{*}, u\right) f_{i}\left(t, \mathbf{x}, \mathbf{v}, u_{*}\right) g_{i r}\left(t, \mathbf{x}, \mathbf{v}, \omega^{*}\right) d u_{*} d \omega^{*} \\
& -f_{i}(t, \mathbf{x}, \mathbf{v}, u) \sum_{r=1}^{m} \eta_{i r}^{e} \int_{D_{u}} g_{i r}\left(t, \mathbf{x}, \mathbf{v}, \omega^{*}\right) d \omega^{*}
\end{aligned}
$$

where $g_{i}=\left(g_{i 1}, \ldots, g_{i r}\right)$ and

$-\eta_{i r}^{e}$ is the inner-outer encounter rate between the $r$ th external agent, with state $\omega^{*}$, and the cell of the $i$ th population, with state $u_{*}$.

- $\mathcal{B}_{i r}\left(u_{*}, \omega^{*}, u\right)$ is the inner-outer transition probability density which describes the probability density that a cell of the $i$ th population, with state $u_{*}$, falls into the state $u$ after an interaction with the $r$ th external agent whose state is $\omega^{*}$.

The density $\mathcal{B}_{i r}$ satisfies, for all $r \in\{1,2, \ldots, m\}$ and $i \in\{1,2, \ldots, n\}$, the following condition:

$$
\int_{D_{u}} \mathcal{B}_{i r}\left(u_{*}, \omega^{*}, u\right) d u=1, \quad \forall u_{*}, \omega^{*} \in D_{u} .
$$

Bearing all above in mind, the thermostatted kinetic framework for open systems with proliferative/destructive and mutative interactions reads:

$$
\left(\partial_{t}+\mathbf{v} \cdot \nabla_{\mathbf{x}}\right) f_{i}+\mathcal{T}_{F_{i}}[\mathbf{f}]=J_{i}[\mathbf{f}]+N_{i}[\mathbf{f}]+M_{i}[\mathbf{f}]+Q_{i}\left[\mathbf{f}, g_{i}\right]+v V_{i}\left[f_{i}\right] .
$$

The thermostatted framework (2.11) is the underlying framework for the derivation of the related macroscopic equation by the low-field scaling. In particular solutions of (2.11) are assumed to be bounded in a space of functions where all needed convergence results will be true.

\section{Macroscopic Quantities and Turning Operator}

This section is devoted to the definition of the local macroscopic quantities and the derivation of the main properties of the turning operator related to the velocity-jump process that will be used in the next section.

Bearing the thermostatted framework (2.11) in mind, the local macroscopic quantities, for $i \in\{1,2, \ldots, n\}$, can be defined as follows:

- The local density $\rho\left[f_{i}\right](t, \mathbf{x}, u)$ of the $i$ th functional subsystem defined at time $t$ in the position $\mathbf{x}$ and activity $u$, reads:

$$
\rho_{i}:=\rho\left[f_{i}\right](t, \mathbf{x}, u)=\int_{D_{\mathbf{v}}} f_{i}(t, \mathbf{x}, \mathbf{v}, u) d \mathbf{v}
$$

- The relative mass velocity of particles $\mathbb{U}_{i}(t, \mathbf{x}, u)$ defined on $\left[0, \infty\left[\times D_{\mathbf{x}} \times D_{u}\right.\right.$, reads:

$$
\mathbb{U}_{i}:=\mathbb{U}_{i}(t, \mathbf{x}, u)=\frac{1}{\rho\left[f_{i}\right](t, \mathbf{x}, u)} \int_{D_{\mathbf{v}}} \mathbf{v} f_{i}(t, \mathbf{x}, \mathbf{v}, u) d \mathbf{v} .
$$

- The pressure term reads:

$$
\mathbb{P}_{i}:=\mathbb{P}_{i}(t, \mathbf{x}, u)=\int_{D_{\mathbf{v}}}\left(\mathbf{v}-\mathbb{U}_{i}\right) \otimes\left(\mathbf{v}-\mathbb{U}_{i}\right) f_{i}(t, \mathbf{x}, \mathbf{v}, u) d \mathbf{v} .
$$

- The local linear activation moment at time $t$ in $\mathbf{x}$ reads:

$$
\mathbb{A}\left[f_{i}\right](t, \mathbf{x})=\int_{\Sigma} u f_{i}(t, \mathbf{x}, \mathbf{v}, u) d \mathbf{v} d u
$$

where $\Sigma=D_{\mathbf{v}} \times D_{u}$.

Note that, by definition of $\mathbb{U}$, one has

$$
\int_{D_{\mathbf{v}}} \mathbf{v} \otimes \mathbf{v} f_{i}(t, \mathbf{x}, \mathbf{v}, u) d \mathbf{v}=\rho_{i} \mathbb{U}_{i} \otimes \mathbb{U}_{i}+\mathbb{P}_{i} .
$$

Macroscopic gross quantities can be easily obtained by summing in the local quantities with respect to $i$. 
The Hilbert space $L^{2}\left(D_{\mathbf{v}}, d \mathbf{v}\right)$ endowed with the usual scalar product

$$
\langle f, g\rangle=\int_{D_{\mathbf{v}}} f(\mathbf{v}) g(\mathbf{v}) d \mathbf{v}, \quad f, g \in L^{2}\left(D_{\mathbf{v}}, d \mathbf{v}\right)
$$

will be used in the sequel and the average of the function $\varphi$ with respect to variable $\mathbf{v}$ will be denoted by

$$
\langle\varphi\rangle:=\langle\varphi, 1\rangle=\int_{D_{\mathbf{v}}} \varphi(\mathbf{v}) d \mathbf{v} .
$$

Moreover the following Kronecker delta will be used:

$$
\delta_{i j}=\left\{\begin{array}{l}
1 \text { if } i=j \\
0 \text { if } i \neq j
\end{array}\right.
$$

Preliminary to the low-field limit are the properties of the turning operator $V_{i}\left[f_{i}\right]$, which are summarized in the following lemma, see [33] for a detailed proof.

\section{Lemma 1 Assume that:}

$\left(\boldsymbol{A}_{1}\right)\left\|V_{i}\left[f_{i}\right]\right\|_{L^{1}\left(D_{\mathbf{v}}, d \mathbf{v}\right)}=\left\|\mathbf{v} V_{i}\left[f_{i}\right]\right\|_{L^{1}\left(D_{\mathbf{v}}, d \mathbf{v}\right)}=0, \forall \mathbf{x} \in D_{\mathbf{x}}, u \in D_{u}$.

$\left(\boldsymbol{A}_{2}\right)$ There exists a bounded equilibrium velocity distribution $G_{i}(\mathbf{v}): D_{\mathbf{v}} \rightarrow \mathbb{R}^{+}$, independent of $t$ and $\mathbf{x}$, such that:

$$
T_{i}\left(\mathbf{v}^{*}, \mathbf{v}\right) G_{i}(\mathbf{v})=T_{i}\left(\mathbf{v}, \mathbf{v}^{*}\right) G_{i}\left(\mathbf{v}^{*}\right) .
$$

and $\left\|\mathbf{v} G_{i}(\mathbf{v})\right\|_{L^{1}\left(D_{\mathbf{v}}, d \mathbf{v}\right)}=0,\left\|G_{i}(\mathbf{v})\right\|_{L^{1}\left(D_{\mathbf{v}}, d \mathbf{v}\right)}=0, \forall \mathbf{x} \in D_{\mathbf{x}}, u \in D_{u}$.

$\left(\boldsymbol{A}_{3}\right)$ The kernel $T_{i}\left(\mathbf{v}, \mathbf{v}^{*}\right)$ is bounded, and there exists a constant $\sigma_{i}>0$ such that

$$
T_{i}\left(\mathbf{v}, \mathbf{v}^{*}\right) \geq \sigma_{i} G_{i}(\mathbf{v}), \quad \forall\left(\mathbf{v}, \mathbf{v}^{*}\right) \in D_{\mathbf{v}} \times D_{\mathbf{v}} .
$$

Then

(i) (H-Theorem). The entropy equality holds

$$
-\int_{D_{\mathbf{v}}} V_{i}\left[f_{i}\right] f_{i} F_{i}^{-1} d \mathbf{v}=\frac{1}{2} \int_{D_{\mathbf{v}} \times D_{\mathbf{v}}} T_{i}\left(\mathbf{v}, \mathbf{v}^{*}\right) F_{i}^{*}\left(\frac{f_{i}^{*}}{F_{i}^{*}}-\frac{f_{i}}{F_{i}}\right)^{2} d \mathbf{v} d \mathbf{v}^{*} \geqslant 0
$$

(ii) The null-space of $V_{i}$ is spanned by a unique normalized and nonnegative function $F_{i}(\mathbf{v})$ :

$$
\operatorname{Ker}\left(V_{i}\right)=\operatorname{Span}\left\{F_{i}\right\}
$$

(iii) There exists a constant $\kappa>0$ such that

$$
-\int_{D_{\mathbf{v}}} V_{i}\left[f_{i}\right] f_{i} F_{i}^{-1} d \mathbf{v} \geqslant \kappa \int_{D_{\mathbf{v}}}\left|f_{i}-\left\langle f_{i}\right\rangle F_{i}\right|^{2} \frac{\mathrm{v} d \mathbf{v}}{F_{i}(\mathbf{v})}=\kappa\left\|f_{i}-\left\langle f_{i}\right\rangle\right\|_{H}^{2}
$$

being $\langle g\rangle:=\int_{D_{\mathbf{v}}} g d \mathbf{v}$

(iv) For any $h_{i} \in H$ satisfying $\int_{D_{\mathbf{v}}} h_{i} d \mathbf{v}=0$, there exists a unique $f_{i} \in H$ such that $V_{i}\left[f_{i}\right]=h_{i}$ and $\int_{D_{\mathbf{v}}} f_{i} d \mathbf{v}=0$.

\section{The Macroscopic Framework at Tissue Scale}

This section deals with the low-field limit of the thermostatted kinetic framework (2.11). In particular the macroscopic equation at the tissue scale is derived according to the following low-field scaling

$$
t \rightarrow \frac{t}{\varepsilon}, \quad \mathcal{F}_{i} \rightarrow \varepsilon^{\ell} \mathcal{F}_{i} \quad \ell \geq 1
$$

with the following rate choice:

$$
\eta=\varepsilon^{r}, \quad \mu=\varepsilon^{q}, \quad \varphi=\varepsilon^{m}, \quad \nu=\frac{1}{\varepsilon^{s}}, \quad \eta^{e}=\varepsilon^{h}
$$


where the numbers $h, m, q, r, s \geq 1$ are chosen according to biological requests. Therefore the distribution function of the $i$ th functional subsystem and the distribution function of the irth external agent are rescaled as follows:

$$
f_{i}^{\varepsilon}(t, \mathbf{x}, \mathbf{v}, u)=f_{i}\left(\frac{t}{\varepsilon}, \mathbf{x}, \mathbf{v}, u\right), \quad g_{i r}^{\varepsilon}(t, \mathbf{x}, \mathbf{v}, u)=g_{i r}\left(\frac{t}{\varepsilon}, \mathbf{x}, \mathbf{v}, u\right) .
$$

Let $\mathbf{f}^{\varepsilon}=\left(f_{1}^{\varepsilon}, f_{2}^{\varepsilon}, \ldots, f_{n}^{\varepsilon}\right)$ be the vector of the rescaled distribution functions and $g_{i}^{\varepsilon}=\left(g_{i 1}^{\varepsilon}, g_{i 2}^{\varepsilon}, \ldots, g_{i r}^{\varepsilon}\right)$, then the rescaled thermostatted framework (2.11) reads:

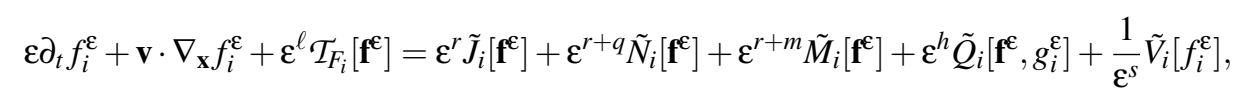

where $\tilde{J}_{i}\left[\mathbf{f}^{\mathcal{\varepsilon}}\right], \tilde{N}_{i}\left[\mathbf{f}^{\mathfrak{\varepsilon}}\right], \tilde{M}_{i}\left[\mathbf{f}^{\mathfrak{⿷}}\right], \tilde{V}_{i}\left[f_{i}^{\mathcal{\varepsilon}}\right], \tilde{Q}_{i}\left[\mathbf{f}^{\mathcal{\varepsilon}}, g_{i}^{\varepsilon}\right]$, and $\mathcal{T}_{F_{i}}\left[\mathbf{f}^{\mathfrak{\varepsilon}}\right]$ are the rescaled conservative, nonconservative, mutating, turning, external agents and thermostat operators, respectively, that read:

$$
\begin{gathered}
\tilde{J}_{i}\left[\mathbf{f}^{\varepsilon}\right]=\sum_{j=1}^{n} \int_{D_{u} \times D_{u}} \mathcal{A}_{i j}\left(u_{*}, u^{*}, u\right) f_{i}^{\varepsilon}\left(t, \mathbf{x}, \mathbf{v}, u_{*}\right) f_{j}^{\varepsilon}\left(t, \mathbf{x}, \mathbf{v}, u^{*}\right) d u_{*} d u^{*}-\sum_{j=1}^{n} f_{i}^{\varepsilon}(t, \mathbf{x}, \mathbf{v}, u) \int_{D_{u}} f_{j}^{\varepsilon}\left(t, \mathbf{x}, \mathbf{v}, u^{*}\right) d u^{*} \\
\tilde{N}_{i}\left[\mathbf{f}^{\varepsilon}\right]=f_{i}^{\varepsilon}(t, \mathbf{x}, \mathbf{v}, u) \sum_{j=1}^{n} \int_{D_{u}} f_{j}^{\varepsilon}\left(t, \mathbf{x}, \mathbf{v}, u^{*}\right) d u^{*} \\
\tilde{M}_{i}\left[\mathbf{f}^{\varepsilon}\right]=\sum_{h=1}^{n} \sum_{k=1}^{n} \int_{D_{u} \times D_{u}} f_{h}^{\varepsilon}\left(t, \mathbf{x}, \mathbf{v}, u_{*}\right) f_{k}^{\varepsilon}\left(t, \mathbf{x}, \mathbf{v}, u^{*}\right) d u_{*} d u^{*}, \\
\tilde{V}_{i}\left[f_{i}^{\varepsilon}\right]=\int_{D_{\mathbf{v}}}\left[T_{i}\left(\mathbf{v}^{*}, \mathbf{v}\right) f_{i}^{\varepsilon}\left(t, \mathbf{x}, \mathbf{v}^{*}, u\right)-T_{i}\left(\mathbf{v}, \mathbf{v}^{*}\right) f_{i}^{\varepsilon}(t, \mathbf{x}, \mathbf{v}, u)\right] d \mathbf{v}^{*} \\
\tilde{Q}_{i}\left[\mathbf{f}^{\varepsilon}, g_{i}^{\varepsilon}\right]=\sum_{r=1}^{m} \int_{D_{u} \times D_{u}} \mathcal{B}_{i r}\left(u_{*}, \omega^{*}, u\right) f_{i}^{\varepsilon}\left(t, \mathbf{x}, \mathbf{v}, u_{*}\right) g_{i r}^{\varepsilon}\left(t, \mathbf{x}, \mathbf{v}, \omega^{*}\right) d u_{*} d \omega^{*}-f_{i}^{\varepsilon}(t, \mathbf{x}, \mathbf{v}, u) \sum_{r=1}^{m} \int_{D_{u}} g_{i r}^{\varepsilon}\left(t, \mathbf{x}, \mathbf{v}, \omega^{*}\right) d \omega^{*}, \\
\mathcal{T}_{F_{i}}\left[\mathbf{f}^{\varepsilon}\right]=\partial_{u}\left(F_{i}(u)\left(1-u \int_{\Omega} u \tilde{f}^{\varepsilon} d \mathbf{x} d \mathbf{v} d u\right) f_{i}^{\varepsilon}(t, \mathbf{x}, \mathbf{v}, u)\right)
\end{gathered}
$$

The following lemma hold true.

Lemma 2 Let $f_{i}^{\varepsilon}(t, \mathbf{x}, \mathbf{v}, u)$ be a sequence of solutions of the rescaled thermostatted kinetic equation (4.23). Assume that the turning operator $V_{i}$ satisfies the assumptions $\left(\boldsymbol{A}_{1}-\boldsymbol{A}_{2}-\boldsymbol{A}_{3}\right)$ and

$$
\begin{aligned}
f_{i}^{\varepsilon} & \underset{\varepsilon \rightarrow 0}{\longrightarrow} f_{i} \quad \text { a.e. in }[0, \infty) \times D_{\mathbf{x}} \times D_{\mathbf{v}} \times D_{u}, \\
g_{i r}^{\varepsilon} & \underset{\varepsilon \rightarrow 0}{\longrightarrow} g_{i r} \\
\tilde{V}_{i}\left[f_{i}^{\varepsilon}\right] & \underset{\varepsilon \rightarrow 0}{\longrightarrow} \tilde{V}_{i}\left[f_{i}\right]
\end{aligned}
$$

Then the asymptotic limit $f_{i}$ of the sequence $f_{i}^{\varepsilon}$ (modulo the extraction of a subsequence) admits the following factorization:

$$
f_{i}(t, \mathbf{x}, \mathbf{v}, u)=\rho_{i}(t, \mathbf{x}, u) G_{i}(\mathbf{v}),
$$

where $\rho_{i}$ is the local macroscopic density (3.12) of the ith functional subsystem. Moreover

$$
\frac{1}{\varepsilon}\left\langle\mathbf{v} \cdot \nabla_{\mathbf{x}} f_{i}^{\varepsilon}\right\rangle \underset{\varepsilon \rightarrow 0}{\longrightarrow} \delta_{s, 1} \operatorname{div}_{\mathbf{x}}\left\langle\left(\chi_{i}(\mathbf{v}) \otimes \mathbf{v}\right) \nabla_{\mathbf{x}} \rho_{i}\right\rangle
$$

where $\chi_{i}(\mathbf{v})$ is the only solution of the equation $\tilde{V}_{i}\left[f_{i}\right]=\mathbf{v} G_{i}(\mathbf{v})$.

Proof The first part of the Lemma is gained by multiplying equation (4.23) by $\varepsilon^{s}$, letting $\varepsilon$ goes to zero and considering Lemma 1. The limit of the transport term is gained by considering the following equality:

$$
\begin{aligned}
\frac{1}{\varepsilon}\left\langle\mathbf{v} \cdot \nabla_{\mathbf{x}} f_{i, \varepsilon}\right\rangle & =\nabla_{\mathbf{x}} \cdot\left\langle\frac{\mathbf{v} f_{i, \varepsilon}}{\varepsilon}\right\rangle \\
& =\frac{1}{\varepsilon} \operatorname{div}_{\mathbf{x}}\left\langle\mathbf{v} f_{i, \varepsilon} \frac{G_{i}(\mathbf{v})}{G_{i}(\mathbf{v})}\right\rangle=\operatorname{div}_{\mathbf{x}}\left\langle\frac{V_{i}\left[f_{i, \varepsilon}\right]}{\varepsilon} \frac{\chi_{i}(\mathbf{v})}{G_{i}(\mathbf{v})}\right\rangle,
\end{aligned}
$$

and Lemma 1 again. 
The main result of this paper follows.

Theorem 1 Let $f_{i}^{\varepsilon}(t, \mathbf{x}, \mathbf{v}, u)$ be a sequence of solutions of the rescaled thermostatted kinetic equation (4.23). Assume that the turning operator $V_{i}$ satisfies the assumptions $\left(\boldsymbol{A}_{1}-\boldsymbol{A}_{2}-\boldsymbol{A}_{3}\right)$ and

$$
\begin{aligned}
f_{i}^{\varepsilon} & \underset{\varepsilon \rightarrow 0}{\longrightarrow} f_{i} \quad \text { a.e. in }[0, \infty) \times D_{\mathbf{x}} \times D_{\mathbf{v}} \times D_{u}, \\
g_{i}^{\varepsilon} & \underset{\varepsilon \rightarrow 0}{\longrightarrow} g_{i} \\
\tilde{V}_{i}\left[f_{i}^{\varepsilon}\right] & \underset{\varepsilon \rightarrow 0}{\longrightarrow} \tilde{V}_{i}\left[f_{i}\right]
\end{aligned}
$$

Furthermore, assume that

$\left(\boldsymbol{A}_{4}\right)$ The following quantities

$$
\left\langle f_{i}^{\varepsilon}\right\rangle,\left\langle\mathbf{v} f_{i}^{\varepsilon}\right\rangle,\left\langle\mathbf{v} \otimes \mathbf{v} f_{i}^{\varepsilon}\right\rangle,
$$

converge, in the sense of distributions on $\mathbb{R}_{+}^{*} \times D_{\mathbf{x}} \times D_{u}$, to the corresponding quantities

$$
\left\langle f_{i}\right\rangle,\left\langle\mathbf{v} f_{i}\right\rangle,\left\langle\mathbf{v} \otimes \mathbf{v} f_{i}\right\rangle,
$$

(A) The following quantities

$$
\left\langle\tilde{J}_{i}\left[\mathbf{f}^{\mathfrak{\varepsilon}}\right]\right\rangle,\left\langle\tilde{N}_{i}\left[\mathbf{f}^{\mathfrak{\varepsilon}}\right]\right\rangle,\left\langle\tilde{M}_{i}\left[\mathbf{f}^{\mathfrak{⿷}}\right]\right\rangle,\left\langle\tilde{Q}_{i}\left[\mathbf{f}^{\mathfrak{⿷}}, g_{i}^{\varepsilon}\right]\right\rangle,\left\langle\mathcal{T}_{F_{i}}\left[\mathbf{f}^{\mathfrak{\varepsilon}}\right]\right\rangle
$$

converge, in the sense of distributions on $\mathbb{R}_{+}^{*} \times D_{\mathbf{x}} \times D_{u}$, to the corresponding quantities

$$
\left\langle\tilde{J}_{i}[\mathbf{f}]\right\rangle,\left\langle\tilde{N}_{i}[\mathbf{f}]\right\rangle,\left\langle\tilde{M}_{i}[\mathbf{f}]\right\rangle,\left\langle\tilde{Q}_{i}\left[\mathbf{f}, g_{i}\right]\right\rangle,\left\langle\mathcal{T}_{F_{i}}[\mathbf{f}]\right\rangle,
$$

(A) The following quantities

$$
\left\langle\mathbf{v} \tilde{J}_{i}\left[\mathbf{f}^{\mathbf{f}}\right]\right\rangle,\left\langle\mathbf{v} \tilde{N}_{i}\left[\mathbf{f}^{\mathfrak{⿷}}\right]\right\rangle,\left\langle\mathbf{v} \tilde{M}_{i}\left[\mathbf{f}^{\varepsilon}\right]\right\rangle,\left\langle\mathbf{v} \tilde{Q}_{i}\left[\mathbf{f}^{\mathfrak{\varepsilon}}, g_{i}^{\varepsilon}\right]\right\rangle,\left\langle\mathbf{v} \mathcal{T}_{F_{i}}\left[\mathbf{f}^{\mathbf{f}}\right]\right\rangle,
$$

converge, in the sense of distributions on $\mathbb{R}_{+}^{*} \times D_{\mathbf{x}} \times D_{u}$, to the corresponding quantities

$$
\left\langle\mathbf{v} \tilde{I}_{i}[\mathbf{f}]\right\rangle,\left\langle\mathbf{v} \tilde{N}_{i}[\mathbf{f}]\right\rangle,\left\langle\mathbf{v} \tilde{M}_{i}[\mathbf{f}]\right\rangle,\left\langle\mathbf{v} \tilde{Q}_{i}\left[\mathbf{f}, g_{i}\right]\right\rangle,\left\langle\mathbf{v} \mathcal{T}_{F_{i}}[\mathbf{f}]\right\rangle,
$$

and every formally small term in $\varepsilon$ vanishes, then the local macroscopic density $\rho_{i}$ of the ith functional subsystem is the weak solution of the following equation

$$
\begin{aligned}
\partial_{t} \rho_{i}+\delta_{\ell, 1} \partial_{u}\left(F_{i}(u)(1-u \mathbb{A}[\rho](t)) \rho_{i}\right) & =\delta_{s, 1} \operatorname{div}_{\mathbf{x}}\left(\mathbb{D}_{\rho_{i}} \cdot \nabla_{\mathbf{x}} \rho_{i}\right)+\delta_{r, 1} H_{i}[\rho] \\
& +\delta_{r+q, 1} S_{i}[\rho]+\delta_{r+m, 1} L[\rho] \\
& +\delta_{h, 1} W_{i}[\rho]
\end{aligned}
$$

where $\rho=\left(\rho_{1}, \rho_{2}, \ldots, \rho_{n}\right)$ and

$-\mathbb{A}[\rho](t)$ is the following operator:

$$
\mathbb{A}[\rho](t)=\sum_{j=1}^{n} \int_{D_{\mathbf{x}} \times D_{u}} u \rho_{j}(t, \mathbf{x}, u) d \mathbf{x} d u=\int_{D_{\mathbf{x}} \times D_{u}} u \rho(t, \mathbf{x}, u) d \mathbf{x} d u,
$$

$-\mathbb{D}_{\rho_{i}}$ is the following tensor:

$$
\mathbb{D}_{\rho_{i}}=-\int_{D_{\mathbf{v}}} \mathbf{v} \otimes \chi_{i}(\mathbf{v}) d \mathbf{v}
$$

$-H_{i}[\rho](t, \mathbf{x}, u)$ is the following operator:

$$
\begin{aligned}
H_{i}[\rho] & =\sum_{j=1}^{n}\left\langle G_{i}(\mathbf{v}), G_{j}(\mathbf{v})\right\rangle \int_{D_{u} \times D_{u}} \mathcal{A}_{i j}\left(u_{*}, u^{*}, u\right) \rho_{i}\left(t, \mathbf{x}, u_{*}\right) \rho_{j}\left(t, \mathbf{x}, u^{*}\right) d u_{*} d u^{*} \\
& -\rho_{i}(t, \mathbf{x}, u) \sum_{j=1}^{n}\left\langle G_{i}(\mathbf{v}), G_{j}(\mathbf{v})\right\rangle \int_{D_{u}} \rho_{j}\left(t, \mathbf{x}, u^{*}\right) d u^{*}
\end{aligned}
$$

$-S_{i}[\rho](t, \mathbf{x}, u)$ is the following operator:

$$
S_{i}[\boldsymbol{\rho}]=\rho_{i}(t, \mathbf{x}, u) \sum_{j=1}^{n}\left\langle G_{i}(\mathbf{v}), G_{j}(\mathbf{v})\right\rangle \int_{D_{u}} \rho_{j}\left(t, \mathbf{x}, u^{*}\right) d u^{*} .
$$


$-L[\rho](t, \mathbf{x})$ is the following operator:

$$
\begin{aligned}
L[\rho] & =\sum_{h=1}^{n} \sum_{k=1}^{n}\left\langle G_{h}(\mathbf{v}), G_{k}(\mathbf{v})\right\rangle\left(\int_{D_{u}} \rho_{h}\left(t, \mathbf{x}, u_{*}\right) d u_{*}\right) \\
& \times\left(\int_{D_{u}} \rho_{k}\left(t, \mathbf{x}, u^{*}\right) d u^{*}\right) .
\end{aligned}
$$

$-W_{i}[\rho](t, \mathbf{x}, u)$ is the following operator:

$$
\begin{aligned}
W_{i}[\rho] & =\sum_{r=1}^{m} \int_{D_{u} \times D_{u}} \mathcal{B}_{i r}\left(u_{*}, \omega^{*}, u\right) \rho_{i}\left(t, \mathbf{x}, u_{*}\right)\left\langle G_{i}(\mathbf{v}), g_{i r}\left(t, \mathbf{x}, \mathbf{v}, \omega^{*}\right)\right\rangle d u_{*} d \omega^{*} \\
& -\rho_{i}(t, \mathbf{x}, u) \sum_{r=1}^{m} \int_{D_{u}}\left\langle G_{i}(\mathbf{v}), g_{i r}\left(t, \mathbf{x}, \mathbf{v}, \omega^{*}\right)\right\rangle d \omega^{*}
\end{aligned}
$$

Proof Assume that $\ell>1, r>1, q>1, m>1$, and $h>1$. Considering the average of the rescaled thermostatted framework equation (4.23) with respect to $\mathbf{v}$, using the assumption $\mathbf{A}_{1}$ and dividing by $\varepsilon$, one obtains:

$$
\partial_{t}\left\langle f_{i}^{\varepsilon}\right\rangle+\frac{1}{\varepsilon}\left\langle\mathbf{v} \cdot \nabla_{\mathbf{x}} f_{i}^{\varepsilon}\right\rangle+\varepsilon^{\ell-1}\left\langle\mathcal{T}_{F_{i}}\left[\mathbf{f}^{\mathfrak{E}}\right]\right\rangle=\tilde{Z}\left[\mathbf{f}^{\mathfrak{\ell}}\right]
$$

where

$$
\tilde{Z}\left[\mathbf{f}^{\varepsilon}\right]=\varepsilon^{r-1}\left\langle\tilde{J}\left[\mathbf{f}^{\mathfrak{f}}\right]\right\rangle+\varepsilon^{r+q-1}\left\langle\tilde{N}\left[\mathbf{f}^{\varepsilon}\right]\right\rangle+\varepsilon^{r+m-1}\left\langle\tilde{M}\left[\mathbf{f}^{\mathfrak{\varepsilon}}\right]\right\rangle+\varepsilon^{h-1}\left\langle\tilde{Q}_{i}\left[\mathbf{f}^{\varepsilon}, g_{i}^{\varepsilon}\right]\right\rangle
$$

When $\varepsilon \rightarrow 0$, the term $\tilde{Z}\left[\mathbf{f}^{\varepsilon}\right]$ goes to zero, and by using Lemma 2 we have the limit (4.34).

Then the asymptotic limit of the thermostat term reads:

$$
\begin{aligned}
\varepsilon^{\ell-1}\left\langle\mathcal{T}_{F_{i}}\left[\mathbf{f}^{\varepsilon}\right]\right\rangle & =\varepsilon^{\ell-1}\left\langle\partial_{u}\left(F_{i}(u)\left(1-u \int_{\Omega} u \tilde{f}^{\varepsilon} d \Omega\right) f_{i}^{\varepsilon}\right)\right\rangle \\
\underset{\varepsilon \rightarrow 0}{\longrightarrow} & \delta_{l, 1}\left\langle\partial_{u}\left(F_{i}(u)\left(1-u \sum_{j=1}^{n} \int_{\Omega} u \rho_{j} G_{j} d \Omega\right) \rho_{i} G_{i}\right)\right\rangle \\
& =\delta_{l, 1} \partial_{u}\left(F_{i}(u)(1-u \mathbb{A}[\rho](t)) \rho_{i}\right)
\end{aligned}
$$

Then, it is an easy task to show that

$$
\begin{aligned}
& \varepsilon^{r-1}\left\langle\tilde{J}_{i}\left[\mathbf{f}^{\mathfrak{\varepsilon}}\right]\right\rangle \underset{\varepsilon \rightarrow 0}{\longrightarrow} \delta_{r, 1} H_{i}[\rho] \\
& \varepsilon^{r+q-1}\left\langle\tilde{N}_{i}\left[\mathbf{f}^{\epsilon}\right]\right\rangle \underset{\varepsilon \rightarrow 0}{\longrightarrow} \delta_{r+q, 1} S_{i}[\rho] \\
& \varepsilon^{r+m-1}\left\langle\tilde{M}_{i}\left[\mathbf{f}^{€}\right]\right\rangle \underset{\varepsilon \rightarrow 0}{\longrightarrow} \delta_{r+m, 1} L[\rho] \\
& \varepsilon^{h-1}\left\langle\tilde{Q}_{i}\left[\mathbf{f}^{\varepsilon}, g_{i}^{\varepsilon}\right]\right\rangle \underset{\varepsilon \rightarrow 0}{\longrightarrow} \delta_{h, 1} W_{i}[\rho]
\end{aligned}
$$

where $H_{i}[\rho], S_{i}[\rho], L[\rho]$ and $W_{i}[\rho]$ are the operators defined in Eqs. (4.42), (4.43), (4.44) and (4.45) respectively. Therefore the proof is concluded.

\subsection{A model for tumor-immune-system competition under the action of a vaccine}

This section deals with the derivation of a mathematical model for tumor-immune-system competition. Since the aim of this section is to show the main steps of the asymptotic method proposed in the present paper, the phenomenological analysis will be limited to the interactions among the following three functional subsystems:

Normal cells Nc, whose distribution function is $f_{1}(t, \mathbf{x}, \mathbf{v}, u)$ and the activity variable $u \in D_{u}=[0,+\infty)$ represents the mutation ability.

Mammary cancer cells $\mathbf{C c}$, whose distribution function is $f_{2}(t, \mathbf{x}, \mathbf{v}, u)$ and the activity variable $u \in D_{u}=[0,+\infty)$ represents the progression towards high state of malignancy. 
Immune system cells ISc, whose distribution function is $f_{3}(t, \mathbf{x}, \mathbf{v}, u)$ and the activity variable $u$ represents the activation.

In particular, we assume the existence of one external action at the microscopic scale that acts on the Cc by reducing their malignancy state; we consider the role of the triplex cellular vaccine, whose distribution function is $g_{21}(t, \mathbf{x}, \mathbf{v}, \omega)$ and the activity variable $\omega \in D_{u}=[0,+\infty)$ represents the ability to stimulate the immune system, see paper [34] for further details.

We assume that inner-inner and outer-inner encounter rates are constants for all interacting cells. Specifically $\eta_{i j}=\eta$ for all $i, j \in$ $\{1,2,3\}$, and $\eta_{21}^{e}=\eta^{e}$. The probability density $\mathcal{A}_{i j}$ and $\mathcal{B}_{21}$ are assumed to be defined by a delta Dirac function (deterministic output $m_{i j}\left(u_{*}, u^{*}\right)$ and $n_{21}\left(u_{*}, \omega^{*}\right)$ of a pair interaction) depending on the microscopic state of the interacting cells:

$$
\begin{aligned}
& \mathcal{A}_{i j}\left(u_{*}, u^{*}, u\right)=\delta\left(u-m_{i j}\left(u_{*}, u^{*}\right)\right), \\
& \mathcal{B}_{21}\left(u_{*}, \omega^{*}, u\right)=\delta\left(u-n_{21}\left(u_{*}, \omega^{*}\right)\right) .
\end{aligned}
$$

Moreover we assume that $D_{\mathbf{v}}$ is the 3 -sphere of radius $R>0$ and we let $D_{\mathbf{x}}$ and $\mathcal{F}_{i}(u)$ arbitrary.

Interactions with transitions of activity. We assume that only Cc are subjected to transitions of activity, then $J_{1}[\mathbf{f}]=J_{3}[\mathbf{f}]=0$. In particular Cc have a tendency to increase their malignancy when they interact with Nc. Specifically we have:

$$
m_{21}\left(u_{*}, u^{*}\right)=u_{*}+\psi
$$

where $\psi$ is a positive parameter related to the ability of Cc to reach high states of malignancy. Accordingly the conservative operator $J_{2}[\mathbf{f}]=J_{2}[\mathbf{f}](t, \mathbf{x}, \mathbf{v}, u)$ reads:

$$
J_{2}[\mathbf{f}]=\eta\left[f_{2}(t, \mathbf{x}, \mathbf{v}, u-\psi)-f_{2}(t, \mathbf{x}, \mathbf{v}, u)\right] \int_{0}^{\infty} f_{1}(t, \mathbf{x}, \mathbf{v}, u) d u .
$$

Interactions with the triplex vaccine. The cellular vaccine produces a decrease in the microscopic state of $\mathrm{Cc}$ towards lower states of malignancy, then

$$
n_{21}\left(u_{*}, \omega^{*}\right)=u_{*}-\psi_{2},
$$

where $\psi_{2}$ is a positive parameter. Assuming that $g_{21}(t, \mathbf{x}, \mathbf{v}, \omega)=a e^{-b \omega}$ (exponential decay with respect to $\omega$ ), $a, b>0$, we have

$$
Q\left[f_{2}, g_{2}\right]=\frac{a}{b} \eta^{e} e^{-b \omega}\left[f_{2}(t, \mathbf{x}, \mathbf{v}, u)-f_{2}(t, \mathbf{x}, \mathbf{v}, u+\psi)\right] .
$$

Interactions with proliferation of cells. We assume that $\mathrm{Nc}$ proliferate when encounter each other, Cc proliferate when encounter Nc and ISc, ISc proliferate when encounter Cc. Then the proliferation rates read:

$$
\begin{aligned}
& \mu_{11}^{p}\left(u, u^{*}\right)=\beta, \\
& \mu_{21}^{p}\left(u, u^{*}\right)=\alpha \beta, \\
& \mu_{23}^{p}\left(u, u^{*}\right)=\alpha \beta, \\
& \mu_{32}^{p}\left(u, u^{*}\right)=\beta_{I},
\end{aligned}
$$

where $\beta>0$ is the proliferation rate of $\mathrm{Nc}, \alpha$ is a scale parameter, and $\beta_{I}$ is the proliferation rate of the ISc. Accordingly the proliferative terms read:

$$
\begin{aligned}
& P_{1}[\mathbf{f}]=\eta \beta f_{1}(t, \mathbf{x}, \mathbf{v}, u) \int_{0}^{\infty} f_{1}(t, \mathbf{x}, \mathbf{v}, u) d u, \\
& P_{2}[\mathbf{f}]=\eta \alpha \beta f_{2}(t, \mathbf{x}, \mathbf{v}, u) \int_{0}^{\infty}\left[f_{1}(t, \mathbf{x}, \mathbf{v}, u)+f_{3}(t, \mathbf{x}, \mathbf{v}, u)\right] d u, \\
& P_{3}[\mathbf{f}]=\eta \beta_{I} f_{3}(t, \mathbf{x}, \mathbf{v}, u) \int_{0}^{\infty} f_{2}(t, \mathbf{x}, \mathbf{v}, u) d u .
\end{aligned}
$$

Interactions with inhibition of cells. We assume that $\mathrm{Nc}$ are killed by $\mathrm{Cc}, \mathrm{Cc}$ are inhibited by ISc, ISc are eliminated by Cc. Then the destruction rates read:

$$
\begin{aligned}
& \mu_{12}^{d}\left(u, u^{*}\right)=\alpha \delta, \\
& \mu_{23}^{d}\left(u, u^{*}\right)=\delta, \\
& \mu_{32}^{d}\left(u, u^{*}\right)=\delta_{I},
\end{aligned}
$$


where $\delta$ is the destruction rate of Cc by ISc and $\delta_{I}$ the destruction rate of the ISc by Mc. Accordingly the destructive terms read:

$$
\begin{aligned}
& D_{1}[\mathbf{f}]=\eta \psi \delta f_{1}(t, \mathbf{x}, \mathbf{v}, u) \int_{0}^{\infty} f_{2}(t, \mathbf{x}, \mathbf{v}, u) d u, \\
& D_{2}[\mathbf{f}]=\eta \delta f_{2}(t, \mathbf{x}, \mathbf{v}, u) \int_{0}^{\infty} f_{3}(t, \mathbf{x}, \mathbf{v}, u) d u, \\
& D_{3}[\mathbf{f}]=\eta \delta_{I} f_{3}(t, \mathbf{x}, \mathbf{v}, u) \int_{0}^{\infty} f_{2}(t, \mathbf{x}, \mathbf{v}, u) d u .
\end{aligned}
$$

Bearing all above in mind, we have:

$$
\mu_{i j}\left(u_{*}, u^{*}\right)=\mu_{i j}^{p}\left(u_{*}, u^{*}\right)-\mu_{i j}^{d}\left(u_{*}, u^{*}\right)
$$

and then

$$
N_{i}[\mathbf{f}](t, \mathbf{x}, \mathbf{v}, u)=P_{i}[\mathbf{f}](t, \mathbf{x}, \mathbf{v}, u)-D_{i}[\mathbf{f}](t, \mathbf{x}, \mathbf{v}, u) .
$$

Interactions with mutation. We assume that $\mathrm{Nc}$ may generate new $\mathrm{Cc}$ when interacting with $\mathrm{Nc}$ and $\mathrm{Cc}$. In particular, we assume that the microscopic state of the entities does not change during the mutation. Then, we have:

$$
\begin{aligned}
& \varphi_{11}^{2}\left(u_{*}, u^{*}, u\right)=\alpha \gamma \delta\left(u-u_{*}\right), \\
& \varphi_{12}^{2}\left(u_{*}, u^{*}, u\right)=\gamma \delta\left(u-u_{*}\right),
\end{aligned}
$$

where $\gamma$ is the mutation rate of $\mathrm{Nc}$ when they encounter $\mathrm{Cc}$. Thus the mutative term reads:

$$
M_{2}[\mathbf{f}]=\eta \gamma f_{1}(t, \mathbf{x}, \mathbf{v}, u)\left(\alpha \int_{0}^{\infty} f_{1}(t, \mathbf{x}, \mathbf{v}, u) d u+\int_{0}^{\infty} f_{2}(t, \mathbf{x}, \mathbf{v}, u) d u\right) .
$$

Velocity-jump process. We assume that $G_{i}(\mathbf{v})=1 /\left|D_{\mathbf{v}}\right|$, then:

$$
T_{i}\left(\mathbf{v}^{*}, \mathbf{v}\right)=\gamma_{i} G_{i}(\mathbf{v})=\frac{\gamma_{i}}{\left|D_{\mathbf{v}}\right|}, \quad 0<\gamma_{i}<\sigma_{i}
$$

Accordingly, the turning operator reads:

$$
V_{i}\left[f_{i}\right](t, \mathbf{x}, \mathbf{v}, u)=\gamma_{i}\left[\rho_{i}(t, \mathbf{x}, u) G_{i}(\mathbf{v})-f_{i}(t, \mathbf{x}, \mathbf{v}, u)\right] .
$$

Let $\Lambda\left[f_{i}, \mathcal{F}_{i}, \mathbf{f}\right]=\Lambda\left[f_{i}, \mathcal{F}_{i}, \mathbf{f}\right](t, \mathbf{x}, \mathbf{v}, u)$ be the following operator

$$
\Lambda\left[f_{i}, \mathcal{F}_{i}, \mathbf{f}\right]=\left(\partial_{t}+\mathbf{v} \cdot \nabla_{\mathbf{x}}\right) f_{i}+\mathcal{T}_{F_{i}}[\mathbf{f}]-V_{i}\left[f_{i}\right]
$$

Bearing all above in mind, the mathematical model at the cellular scale thus reads:

$$
\left\{\begin{aligned}
\Lambda\left[f_{1}, \mathcal{F}_{1}, \mathbf{f}\right]= & \eta\left(\beta \int_{D_{u}} f_{1} d u-\alpha \delta \int_{D_{u}} f_{2} d u\right) f_{1} \\
\Lambda\left[f_{2}, \mathcal{F}_{2}, \mathbf{f}\right]= & \eta\left[f_{2}(t, \mathbf{x}, \mathbf{v}, u-\psi)-f_{2}(t, \mathbf{x}, \mathbf{v}, u)\right] \int_{D_{u}} f_{1} d u \\
& +\eta\left(\alpha \beta\left(\int_{D_{u}}\left[f_{1}+f_{3}\right] d u\right)-\delta \int_{D_{u}} f_{3} d u\right) f_{2} \\
& +\eta \gamma\left(\alpha \int_{D_{u}} f_{1} d u+\int_{D_{u}} f_{2} d u\right) f_{1} \\
& +\frac{a}{b} \eta^{e} e^{-b \omega}\left[f_{2}(t, \mathbf{x}, \mathbf{v}, u)-f_{2}\left(t, \mathbf{x}, \mathbf{v}, u+\psi_{2}\right)\right] \\
\Lambda\left[f_{3}, \mathcal{F}_{3}, \mathbf{f}\right]= & \eta\left(\beta_{I} \int_{D_{u}} f_{2} d u-\delta_{I} \int_{D_{u}} f_{2} d u\right) f_{3}
\end{aligned}\right.
$$

The macroscopic equation at tissue scale. According to Lemma 1 , the function $\chi_{i}(\mathbf{v})$ reads:

$$
\chi_{i}(\mathbf{v})=\frac{\gamma_{i} \rho_{i}-\mathbf{v}}{\gamma_{i}\left|D_{\mathbf{v}}\right|}
$$

The diffusion tensor $\mathbb{D}_{\rho_{i}}$ defined in (4.41) reads:

$$
\mathbb{D}_{\rho_{i}}=\frac{1}{\gamma_{i}} \int_{D_{\mathbf{v}}}\left[\mathbf{v} \otimes \mathbf{v} G_{i}(\mathbf{v})\right] d \mathbf{v}=\frac{1}{\gamma_{i}\left|D_{\mathbf{v}}\right|} \int_{D_{\mathbf{v}}} \mathbf{v} \otimes \mathbf{v} d \mathbf{v}=\frac{R^{2}}{3 \gamma_{i}} \mathbb{I} .
$$


Straightforward computations show that

$$
\operatorname{div}_{\mathbf{x}}\left(\mathbb{D}_{\rho_{i}} \cdot \nabla_{\mathbf{x}} \rho_{i}\right)=\frac{R^{2}}{3 \gamma_{i}} \Delta_{\mathbf{x}} \rho_{i}
$$

and

$$
\left\langle G_{i}(\mathbf{v}), G_{j}(\mathbf{v})\right\rangle=\frac{1}{\left|D_{\mathbf{v}}\right|}, \quad\left\langle G_{i}(\mathbf{v}), g_{1}\left(t, \mathbf{x}, \mathbf{v}, \omega^{*}\right)\right\rangle=a e^{-b \omega} .
$$

Let $\Xi\left[\rho_{i}, F_{i}, \gamma_{i}\right](t, \mathbf{x}, u)$ be the following operator:

$$
\Xi_{l, s}\left[\rho_{i}, F_{i}, \gamma_{i}\right](t, \mathbf{x}, u)=\partial_{t} \rho_{i}+\delta_{l, 1} \partial_{u}\left(F_{i}(u)(1-u \mathbb{A}[\rho](t)) \rho_{i}\right)-\delta_{s, 1} \frac{R^{2}}{3 \gamma_{i}} \Delta_{\mathbf{x}} \rho_{i},
$$

and

$$
L[\rho]=\frac{1}{\left|D_{\mathbf{v}}\right|} \sum_{h=1}^{3} \sum_{k=1}^{3}\left(\int_{D_{u}} \rho_{h}(t, \mathbf{x}, u) d u\right)\left(\int_{D_{u}} \rho_{k}(t, \mathbf{x}, u) d u\right) .
$$

Then the macroscopic model (4.39) related to the evolution of the local densities of the system thus reads:

$$
\left\{\begin{aligned}
\Xi_{l, s}\left[\rho_{1}, F_{1}, \gamma_{1}\right]= & \left(\delta_{r+q, 1}-\delta_{r, 1}\right) \frac{\rho_{1}(t, \mathbf{x}, u)}{\left|D_{\mathbf{v}}\right|} \tilde{\rho}(t, \mathbf{x})+\delta_{r+m, 1} L[\rho](t, \mathbf{x}) \\
\Xi_{l, s}\left[\rho_{2}, F_{2}, \gamma_{2}\right]= & \delta_{r, 1} \frac{\rho_{2}(t, \mathbf{x}, u-\psi)}{\left|D_{\mathbf{v}}\right|} \int_{D_{u}} \rho_{1}(t, \mathbf{x}, u) d u \\
& +\left(\delta_{r+q, 1}-\delta_{r, 1}\right) \frac{\rho_{2}(t, \mathbf{x}, u)}{\left|D_{\mathbf{v}}\right|} \tilde{\rho}(t, \mathbf{x})+\delta_{r+m, 1} L[\rho](t, \mathbf{x}) \\
& +\delta_{h, 1} \frac{a}{b} \eta^{e} e^{-b \omega}\left[\rho_{2}(t, \mathbf{x}, u)-\rho_{2}\left(t, \mathbf{x}, u+\psi_{2}\right)\right] \\
\Xi_{l, s}\left[\rho_{3}, F_{3}, \gamma_{3}\right]= & \left(\delta_{r+q, 1}-\delta_{r, 1}\right) \frac{\rho_{3}(t, \mathbf{x}, u)}{\left|D_{\mathbf{v}}\right|} \tilde{\rho}(t, \mathbf{x})+\delta_{r+m, 1} L[\rho](t, \mathbf{x})
\end{aligned}\right.
$$

where

$$
\tilde{\rho}(t, \mathbf{x})=\sum_{j=1}^{3} \int_{D_{u}} \rho_{j}(t, \mathbf{x}, u) d u
$$

\section{Conclusions}

In this paper, a mathematical framework at the cellular scale has been proposed for the modeling of tumor-immune system competition. The main aim of the paper was the possibility to link the dynamics described at the cellular scale with the dynamics that occurs at macroscopic (tissue) scale. In particular the paper has been focused on the possibility to model the role of external agents, e.g. the environment, a cellular vaccine, that can affect the whole dynamics. The macroscopic equation has been obtained by employing the asymptotic method of the rescaled thermostatted framework under the low-field limit. The main steps of the method have been shown by means of the definition of a specific model.

Looking at the macroscopic equation, it is noticeable that conservative and nonconservative interactions at the cellular scale become source terms for the macroscopic modeling of the tumor tissue growth. Indeed, as the tissue equation shows, different macroscopic phenomena and dynamics can appear depending on the relations among the rates at the cellular scale.

It is worth stressing that, even if this paper can be considered as an important contribution to the multiscale approach, and specifically to the problem of linking the dynamics at the cellular scale with the dynamics at the tissue scale, the problem of linking the dynamics at molecular scale with the dynamics at cellular scale remains elusive and can be considered as a research perspective.

\section{References}

1. S. Schnell, R. Grima, P.K. Maini, American Scientist 95, 134 (2007).

2. A. Zewail, Physical Biology: From Atoms to Medicine, London, UK: Imperial College Press, 2008.

3. B. Di Ventura, C. Lemerle, K. Michalodimitrakis, L. Serrano, Nature 443, 527 (2006).

4. D.C. Walker, J. Southgate, Brief Bioinform 10, 450 (2009).

5. S. Sanga, H.B. Frieboes, X. Zheng, R. Gatenby, E.L. Bearer, V. Cristini, Predictive oncology: a review of multidisciplinary, multiscale in silico modeling linking phenotype, morphology and growth, Neuroimage 37, S120 (2007).

6. R. Eftimie, J.L. Bramson, D.J.D. Earn, Bulletin of Mathematical Biology 73, 2 (2011). 
7. C. Bianca, N. Bellomo, Towards a Mathematical Theory of Multiscale Complex Biological Systems, World Scientific, Series in Mathematical Biology and Medicine, 2011.

8. F. Chiacchio, M. Pennisi, G. Russo, S. Motta, F. Pappalardo, BioMed Research International, 907171 (2014).

9. A. Chauviere, L. Preziosi, and C. Verdier, Eds., Cell Mechanics, CRC Press, Boca Raton, 2010.

10. H. Grad, In "Rarefied Gas Dynamics" (Proc. 3rd Internat. Sympos., Palais de 1'UNESCO, Paris 1962) Vol. 1, $26-59$.

11. C. Bardos, F. Golse and D. Levermore, J. Statist. Phys. 21, 531 (1992).

12. P. L. Lions and N. Masmoudi, Arch. Rational. Mech. Anal. 158, 173 (2001).

13. M. Lachowicz and D.Wrzosek, Math. Models Methods Appl. Sci. 11, 1393 (2001).

14. F. Filbet, P. Laurencot, B. Perthame, J Math Biol. 50, 189 (2005).

15. C. Cercignani, I. Gamba and D. Levermore, Appl. Math. Lett. 4, 111 (1997).

16. F. Poupaud, Z. Angew. Math. Mech. 72, 359 (1992).

17. G. C. Papanicolaou, Bulletin AMS, 81, 330 (1975).

18. T. Hillen, Math. Models Methods Appl. Sci. 12, 1007 (2002).

19. N. Bellomo, A. Bellouquid, J. Nieto, J. Soler, Math. Models Methods Appl. Sci. 21, 1 (2012).

20. F. Poupaud, Z. Angew. Math. Mech. 72, 359 (1992).

21. C. Cercignani, I. Gamba and D. Levermore, Appl. Math. Lett. 4, 111 (1997).

22. J. Nieto, F. Poupaud and J. Soler, Arch. Rational Mech. Anal. 158, 29 (2001).

23. T. Goudon, O. Sánchez, J. Soler and L. L. Bonilla, SIAM J. Appl. Math. 64, 1526 (2004).

24. P. Degond and B. Wennberg, Commun Math Sci. 5, 355 (2007).

25. C. Bianca, Commun Nonlinear Sci Numer Simulat 19, 2155 (2014).

26. C. Bianca, C. Dogbe, and Luca Guerrini, Appl. Math. Inf. Sci. 8, 1 (2014).

27. C. Bianca, J. Riposo, European Physical Journal Plus 130, 159 (2015).

28. C. Bianca, Math Methods Appl Sci 36, 1768 (2013).

29. H. G. Othmer, S. R. Dunbar, W. Alt, J. Math. Biol. 26, 263 (1988).

30. C. Bianca, C. Dogbe, A. Lemarchand, Applied Mathematics and Information Sciences 9, 2477-2484 (2015).

31. G. P. Morris, C.P. Dettmann, Chaos 8, 321 (1998).

32. D. Ruelle, J. Stat. Phys. 95, 393 (1999).

33. C. Bianca, C. Dogbe, Nonlinearity 27 (2014), 2771-2803.

34. C. Bianca, F. Chiacchio, F. Pappalardo, M. Pennisi, BMC Bioinformatics 13, S21 (2012). 